\title{
Fabrication and operation of GRIN probes for in vivo fluorescence cellular imaging of internal organs in small animals
}

\author{
Jun Ki Kim ${ }^{1,4}$, Woei Ming Lee ${ }^{1,4}$, Pilhan Kim ${ }^{1,2,4}$, Myunghwan Choi ${ }^{1,2,4}$, Keehoon Jung ${ }^{1}$, Seonghoon Kim ${ }^{1,2} \&$ \\ Seok Hyun Yun ${ }^{1-3}$
}

\begin{abstract}
${ }^{1}$ Harvard Medical School and Wellman Center for Photomedicine, Massachusetts General Hospital, Boston, Massachusetts, USA. ${ }^{2}$ Graduate School of Nanoscience and Technology (WCU), Korea Advanced Institute of Science and Technology, Daejeon, Korea. ${ }^{3}$ Harvard-MIT Health Sciences and Technology, Cambridge, Massachusetts, USA. ${ }^{4}$ These authors contributed equally to this work. Correspondence should be addressed to S.H.Y. (syun@hms.harvard.edu).
\end{abstract}

Published online 5 July 2012; doi:10.1038/nprot.2012.078

Intravital fluorescence microscopy has emerged as a powerful technique to visualize cellular processes in vivo. However, owing to their size, the objective lenses required have limited physical accessibility to various tissue sites in the internal organs of small animals. The use of small-diameter probes using graded-index (GRIN) lenses expands the capabilities of conventional intravital microscopes to minimally invasive imaging of internal organs. In this protocol, we describe the detailed steps for the fabrication of front- and side-view GRIN probes and the integration and operation of the probes in a confocal microscope to enable visualization of fluorescent cells and microvasculature in various mouse organs. Some experience in building an optical setup is required to complete the protocol. We also present longitudinal imaging of immune cells in renal allografts and tumor development in the colon. Fabrication and integration can be completed in 5-7 h, and a typical in vivo imaging session takes 1-2 $\mathrm{h}$.

\section{INTRODUCTION}

GRIN optical probes for intravital imaging

Intravital fluorescence microscopes have been used to study a variety of cellular-level processes in vivo, such as cell trafficking, intercellular interaction and vascular changes. However, owing to the large size of objective lenses required in these instruments, their applications have been limited mostly to superficial tissues, such as the skin, or to exposed internal organs. To overcome this constraint, endomicroscopy based on miniature optical probes has been developed, providing minimally invasive access to tissues in intact organs ${ }^{1}$. High-quality miniature optical probes can be readily fabricated using commercially available GRIN lenses (Box 1) into dimensions (lengths and diameters) that are suited for minimally invasive high-resolution imaging in small animals ${ }^{2,3}$. A frontview GRIN probe allows in vivo imaging of internal organs after laparotomy ${ }^{2}$. A side-view probe is particularly suited for imaging the mucosa of tubular organs such as gastrointestinal tracts and respiratory airways through natural orifices ${ }^{3}$.

\section{Comparison with other methods}

Advances in micro-optic and fiber-optic technologies has led to the emergence of several robust design strategies for miniature optical probes and commercial instruments from, for example, Pentax ${ }^{4}$, Karl Stortz ${ }^{5}$, Mauna Kea Technologies ${ }^{6}$ and Olympus ${ }^{7}$. The confocal endomicroscope made by Pentax and Optiscan offers spatial resolution similar to a GRIN probe, but the diameter of its scanningfiber probe is relatively large $(>3.5 \mathrm{~mm})$. Microprobe objectives from Olympus have narrower diameters $(1.3-3.5 \mathrm{~mm})$, but the objective has a fixed focal plane that restricts its use in live imaging $^{7}$. The Coloview endoscope from Karl Stortz is optimized for tracking tumor progression in the colon on a macroscopic scale ${ }^{8}$. A handheld flexible fiber-optic probe is commercialized by Mauna Kea Technologies, but its spatial resolution is typically lower than GRIN probes and is confined to the surface of the fiber bundle.
In comparison, a GRIN probe provides microscopic resolution in all three dimensions, which is suitable for cellular studies. Thus, it allows optical sectioning of thick tissues while its mechanical rigidity gives good positional stability during imaging of the tissue surface. In addition, the view angle can be altered by attaching a prism mirror to the tip of the probe (e.g., by $90^{\circ}$ for high-resolution side-view imaging of luminal organs). GRIN optical probes, with small diameters $(0.35-1 \mathrm{~mm})$, moderate lengths $(20-100 \mathrm{~mm})$ and high numerical apertures (NA; 0.4-0.6), present themselves as useful tools for minimally invasive imaging of small animal models such as mice.

\section{Limitations of GRIN probes}

As in conventional confocal fluorescence microscopy, the optical penetration depth of a GRIN probe is limited to about $100 \mu \mathrm{m}$ in most soft tissues. The penetration depth may be improved by a factor of two to three by using two-photon imaging and fluorophores emitting fluorescence in the near-infrared region. Although the confocal endomicroscopy described here is well suited for imaging the outer layer of an organ (i.e., the cortex or mucosa), deeper or inner regions (such as the medulla) are not easily accessible by an optical probe without inflicting excess tissue damage. A potential solution to reach deeper tissue is to insert the probe physically into the target site ${ }^{9}$. With careful insertion, the collateral damage to the surrounding tissue can be minimized. Another limitation stems from the mechanical rigidity of the probe, which makes it difficult to reach deep into the intestinal (e.g., the small intestine) and respiratory (e.g., the bronchus) tracts. This limitation may be mitigated with carefully planned surgical methods, such as a feeding tube-like cannula ${ }^{3}$. In addition, the field of view (FOV) of a probe is typically proportional to the diameter of the GRIN lens and is relatively small compared with a standard objective lens. Therefore, a GRIN probe is not suitable for macroscopic imaging. 


\section{Box 1 | Glossary}

Conjugate plane: one spatial point is relayed onto a second conjugate/reciprocal spatial point through a set of optical lenses. For optimal laser beam steering, a $4 f$ conjugate lens system is used to relay the pivot point on the beam steering mirrors onto the center of back pupil plane of the microscope objective lens.

Field of view (FOV): the diameter of the observable area through the optical probe.

Focal plane: the plane, lying on the focal length away from the center of an optical arrangement, in which an image is formed for light focused from infinity.

GRIN lens (Graded-index or gradient-index lens): a cylindrical glass rod in which the refractive index decreases radially with a parabolic function. A GRIN lens is able to focus, collimate and relay optical beams, similarly to a lens waveguide.

Numerical aperture (NA): a dimensionless number that indicates the resolving power of a microscope objective. The number is obtained by multiplying the index of refraction of the medium in front of the microscope objective by the sine of the half-angle over which the system can accept or emit light.

Optical aberration: a distortion in an optical system that reduces the optical performance as compared with an ideal system. They can arise because of the misalignment or physical imperfection of optical components such as lenses and mirrors. Chromatic aberration refers to a shift of focal plane because of a change in wavelength.

Pitch: the length of the GRIN lens within which a single ray will propagate a complete sinusoidal cycle.

Spatial resolution: the shortest distance between two spatially separated points on a specimen that can be resolved by an imaging system as separate entities.

This limitation can be partially overcome by sequentially scanning the probe to acquire a large-area mosaic image.

\section{Experimental design}

Key design parameters for GRIN probes. The optimal design for a GRIN probe is expected to be different depending on the specific target tissue to image. The key design parameters are the viewing direction, physical dimensions and imaging parameters such as FOV and resolution. In terms of the viewing direction, the probes can be categorized into the front-view and side-view types. In Figure 1, we illustrate the light paths in front-view and side-view probes, each of which comprises three GRIN lenses ${ }^{10}$ : a coupling lens (CL), a relay lens (RL) and an imaging lens (IL). The side-view probe has a prism mirror at the end tip. Generally, the

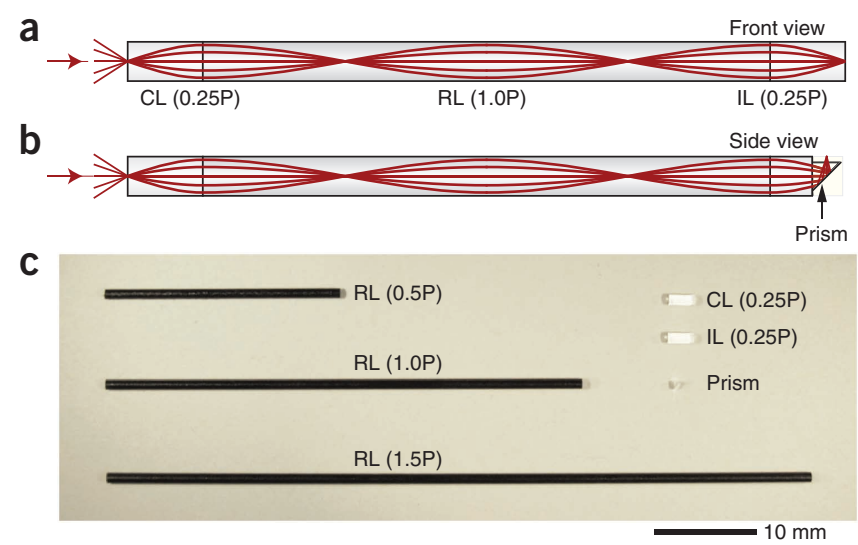

Figure 1 | Schematic of GRIN optical probes. (a) A front-view optical probe. A 0.25 -pitch (P) CL collects light at the input (from the left) and collimates it onto an RL (1.0P). The collimated output is directed onto an IL (0.25P), which serves as the imaging lens. Red lines indicate the path that an optical ray takes. As the focus of the incoming light is raster-scanned, the position of the focus after the imaging lens is changed accordingly. (b) A side-view optical probe. Red lines illustrate ray tracing. A prism is attached onto the polished facet of the imaging lens to direct light onto the side. (c) Photograph of individual GRIN lenses and prism. front-view probe is more appropriate for imaging most organs in the abdominal and thoracic cavities and other tissues such as lymph nodes, which can make contact with the end tip of the probe. The side-view probe is better suited to imaging the mucosa of gastrointestinal tracts and respiratory tracts. The general guidelines are summarized in Table 1.

The diameter of the probe should be small enough to allow minimally invasive access to the target tissue. The diameter of commercially available GRIN lenses ranges from 0.35 to $2 \mathrm{~mm}$. The addition of a protective metal sleeve will increase the diameter by about $0.25 \mathrm{~mm}$. The length of the probe must be sufficiently long to reach the target tissue. The primary purpose of the low-NA RL is to allow for sufficient length. The length of the RL is chosen to be a multiple of half pitch (Fig. 1c). However, as the pitch of RL increases, the spatial resolution tends to degrade owing to increasing optical aberration in the lens. In addition, the risk of damaging the probe by excessive bending increases with the length of the probe. Therefore, it is desirable to minimize the pitch of the RL. For imaging mice, the appropriate length of the probe is $20-30 \mathrm{~mm}$ for organs in the abdominal cavity or $60-100 \mathrm{~mm}$ for gastrointestinal tract imaging.

Although the CL has a 0.25 pitch, which collimates the input beam, the IL should have a pitch equal to or less than 0.25 , such that the focus is formed at or just outside the front or side surface

TABLE 1 | General guidelines for the probes.

\begin{tabular}{llcc}
\hline Target organ & $\begin{array}{l}\text { Viewing } \\
\text { direction }\end{array}$ & $\begin{array}{c}\text { Diameter } \\
(\mathbf{m m})\end{array}$ & $\begin{array}{c}\text { Length } \\
(\mathrm{mm})\end{array}$ \\
\hline $\begin{array}{l}\text { Visceral organs (e.g., liver, } \\
\text { spleen, kidney and so on) }\end{array}$ & Front view & $1-2$ & $20-35$ \\
$\begin{array}{l}\text { Gastrointestinal and respi- } \\
\text { ratory tracts (e.g., colon, } \\
\begin{array}{l}\text { esophagus, trachea and } \\
\text { so on) }\end{array}\end{array}$ & Side view & $0.5-1.5$ & $60-100$ \\
\hline
\end{tabular}


PROTOCOL

TABLE 2 | Selection guidelines for the GRIN lenses.

\begin{tabular}{lllll}
\hline Type & Pitch & NA & Modification & Outcome \\
\hline Coupling lens (CL) & 0.25 & $>0.4$ & Higher NA & $\begin{array}{l}\text { Higher throughput of excitation light } \\
\text { Higher spatial resolution } \\
\text { Smaller FOV }\end{array}$ \\
Imaging lens (IL) & $0.23-0.25$ for front view & $>0.4$ & Higher NA & $\begin{array}{l}\text { Higher spatial resolution } \\
\text { Smaller FOV }\end{array}$ \\
& $0.16-0.17$ for side view & $>0.4$ & Higher NA & $\begin{array}{l}\text { Higher spatial resolution } \\
\text { Smaller FOV }\end{array}$ \\
Relay lens (RL) & A multiple of 0.5 & $0.1-0.2$ & Higher NA & $\begin{array}{l}\text { Larger FOV } \\
\text { Shorter length of the probe }\end{array}$ \\
& & & Longer length & $\begin{array}{l}\text { Increased reach range } \\
\text { Increased optical aberration }\end{array}$ \\
\hline
\end{tabular}

of the probe. For the front-view probe, the appropriate pitch for the IL is $0.23-0.25$. For the side-view probe, the IL should have a pitch of $0.16-0.17$ to compensate for the increased optical path in the attached prism mirror. The IL can be purchased at a custom length from a lens manufacturer or, alternatively, prepared by reducing the length of a 0.25-pitch lens by optical polishing (PROCEDURE Steps 7-14). For a side-view probe, a prism that has the slanted surface coated with metal (aluminum or silver for high reflectivity) is attached after the IL to reflect light by $90^{\circ}$. We recommend selecting a prism with a base that can be fit in the diameter of IL to avoid complications in packing (PROCEDURE Steps 17-22). The selection guidelines for GRIN lenses are summarized in Table 2.

The FOV of the GRIN probe shown in Figure $\mathbf{1}$ is approximately equal to the diameter of the lenses multiplied by the ratio of the NA of the RL to the NA of the IL. (The objective lens of the microscope should be chosen such that the imaging system can accommodate the FOV of the probe.) The FOV of the lenses shown in Figure 1 have a diameter of $1 \mathrm{~mm}$. The probes made with these lenses, as described in this protocol, have a FOV of about $250 \mu \mathrm{m}$ (NA of $\mathrm{RL}=0.1$; NA of $\mathrm{IL}=0.4)$. The NA of the IL determines the overall NA of the probe. The diffraction-limited spatial resolution can be expressed as follows: $0.6 \lambda / \mathrm{NA}$ for transverse resolution and $1.1 \lambda / \mathrm{NA}^{2}$ for axial resolution, where $\lambda$ is the emission wavelength. The actual resolution of a GRIN probe tends to be worse than this theoretical limit, because the effective NA of GRIN lenses is degraded by a factor of 1.5-2 owing to the spatial aberrations in the lenses.

Fabrication of GRIN probes. In this protocol, we describe a stepby-step guide for the fabrication of the probe. The fabrication is relatively easy to carry out by individuals with some experience with general optics. However, for those who are less skillful in optical polishing and assembly or who do not have the necessary tools for fabrication, custom-designed optical probes can be purchased from commercial services (e.g., GRINTECH). In this case, this protocol serves as a general guideline for the custom design. The assembly of optical components requires appropriate tools, such as a stereoscope, a pair of tweezers, a UV light source for UV-curing epoxy and a heater for thermal-curing epoxy. For sufficient precision and ease of assembly, we recommend using
Figure 2 | Probe assembly. (a,b) Photographs of workstation and GRIN lens handling. (c-e) Major steps for front-view optical probe assembly. (c) UV glue is dispensed onto the RL before placing the coupling (or imaging) lens onto the RL. (d) Probe after UV curing of the assembly. (e) Final optical probe after adding a metal sleeve and curing of thermal epoxy. $(\mathbf{f}-\mathbf{k})$ Major steps for side-view optical probe assembly. (f) UV glue is dispensed onto the RL before aligning an IL onto the RL. (g) Probe after UV curing of the assembly. (h) Dispensing UV glue onto the IL and aligning a prism to the IL. (i) UV curing of the IL and prism. (j) Dispensing high-temperature epoxy after inserting the metal sleeve. (k) Final optical probe after curing of epoxy. Scale bar (c-k), $1 \mathrm{~mm}$. (l) A photograph of the completed optical probes. Scale bar, $10 \mathrm{~mm}$.
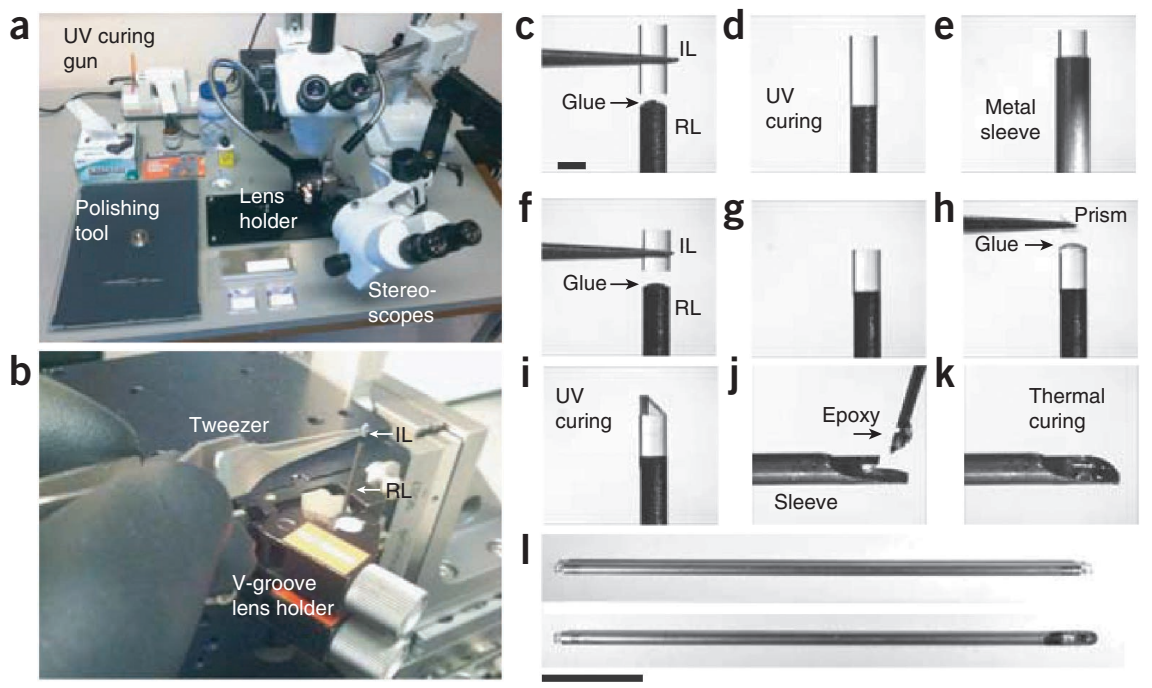
Figure 3 | Front-view probe mount attachment. (a) Front-view probe mount. (b) Individual components: a front-view optical probe (i), front-view probe holder (ii), $x y$ translation stage (iii) and $z$-axis translation stage (iv). (c) Assembly process: complete front-view probe mount (v), microscope objective (vi), $z$-translation stage (vii), cage cube (viii), cage plates (ix) and post (x). The objective lens (vi) of the microscope is attached to an independent $z$-translation stage (vii). The $z$-translation stage is attached to a cage cube (viii) with cage rods, and the probe mount (v) is attached to the cage cube (viii) with cage plates (ix) and a post ( $\mathrm{x}$ ). The focal plane of the probe can be remotely adjusted with the $z$-translation stage (vii) holding the microscope objective (vi), whereas the probe remains fixed in position during imaging. (d) The complete assembly. Front-view optical probe is inserted into the body cavity of an anesthetized mouse, which is held on an $x y z$ stage.
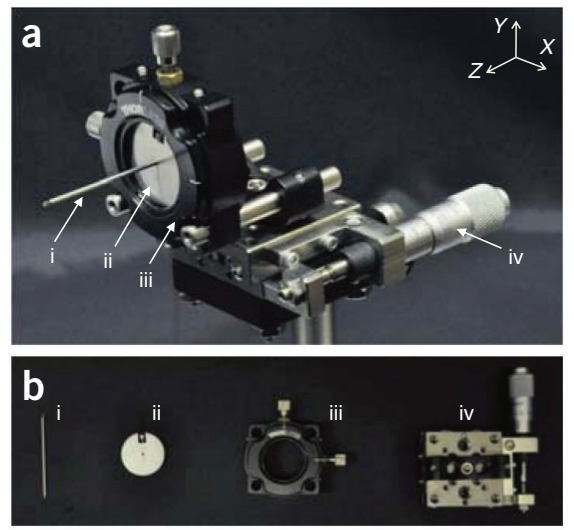

C

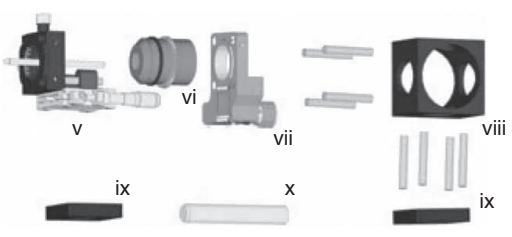

two stereoscopes arranged in orthogonal directions (Fig. 2). In polishing a GRIN lens to a desired length, it is crucial to minimize optical distortion by ensuring that the two end surfaces are flat and parallel. This can be achieved with a manual lapping tool with the help of a custom-made jig (Supplementary Fig. 1). In Figure $2 \mathbf{c}-\mathbf{e}$, we provide a pictorial outline of the fabrication processes for the front-view probe, with photographs taken at three representative steps. For the side-view probe, an additional step is required for the attachment of the prism onto the IL (Fig. 2f-k). It is crucial to avoid using epoxy that tends to emit fluorescence, as it can contribute to the background noise in fluorescence imaging. We recommend using an optical-grade UV-curing epoxy to glue the GRIN lenses. After completing the lens assembly, we recommend using a metal sleeve to reinforce the optical probe, which helps to avoid damage to the optical probe during handling and imaging. The metal sleeve can be purchased from a commercial GRIN lens seller (e.g., GRINTECH and NSG America). After the optical probe is inserted into the metal sleeve, we recommend using a thermal-curing epoxy, which is much more mechanically rigid than UV-curing epoxy, to attach the metal sleeve to the optical probe. It is crucial to ensure that the metal sleeve covers every interface where the lenses and prism are glued.

Integration of GRIN probe to microscope. The fabricated optical probe needs to be integrated into a microscope system. In this protocol, we describe the steps to assemble mechanical mounts for the probe and to connect the mount to a custom-built laserscanning confocal microscope. Detailed designs of mechanical mounts are provided for the front-view (Fig. 3) and side-view probes (Fig. 4). The mechanical mount for the front-view probe should offer the capability of controlling the position of the probe in the $x y z$ directions with the precision of $1-2 \mu \mathrm{m}$. For the sideview probe, an additional capability of rotating the probe by $360^{\circ}$ with the precision of $5^{\circ}$ is required. For the initial use of any optical probe, we recommend that the user measure its optical performance. The FOV, spatial resolution and chromatic aberration can be characterized with commercially available fluorescent beads. In Box 2 and Figure 5, we describe this procedure in detail. For those users who prefer to use a commercial fluorescence microscope (e.g., bright-field, confocal or two-photon microscope), we provide detailed illustrations for an alternative method optimized for a commercial intravital microscope in Box 3 and Figure 6.

Figure 4 | Side-view probe mount attachment. (a) Side-view probe mount. (b) Individual components: a side-view probe (i), side-view probe holder (ii), xy translation stage (iii), $z$-axis translation stage (iv), timing belt (xi), rotation pulley (xii), cage plate with bearing (xiii) and shaft (xiv). The angular position of the side-view probe is controlled with the rotation shaft (xiv). (c) Assembly process: complete side-view probe mount (v), microscope objective (vi), $z$-translation stage (vii), cage cube (viii), cage plates (ix) and post (x). (d) The complete assembly. Side-view optical probe is inserted into the descending colon through the anus of an anesthetized mouse, which is held on an $x y z$ stage. 


\section{Box 2 | Optical characterization of the fabricated probes TIMING $2.5 \mathrm{~h}$}

The FOV and spatial resolution can be measured using fluorescent samples. Chromatic aberration can be quantified using a multicolor fluorescent sample. Detailed mathematical background for obtaining spatial resolution and FOV can be found in the literature $^{13}$. These measurements are recommended for testing the quality of an optical probe before its first use and can be conducted in any order.

\section{Measuring the FOV TIMING 30 min}

It is necessary to measure the FOV to establish an accurate scale bar in the images. For a front-view probe made with 1-mm-diameter SRL and ILW lenses as described in the main PROCEDURE, the FOV will be about $250 \mu \mathrm{m}$. The FOV of the side-view probe described is about 270-280 $\mu \mathrm{m}$.

1. Dilute large fluorescent beads (10-20 $\mu$ m diameter) in water $(0.1-1 \%$, vol/vol), dispense $10 \mu$ on a coverslip and air-dry the coverslip for 20 min.

2. Position the dried sample on the focal plane of the optical probe (Step 24 of the main PROCEDURE).

3. Pick a fluorescent bead in the view, move the stage laterally to position the fluorescent bead at the edge of the view, and then record the current position of the stage.

4. Move the stage in one direction, either $x$ or $y$, to reposition the fluorescent bead to the edge in the opposing direction. Record the position of the stage.

5. Calculate the difference between the positions to measure the FOV.

6. Alternatively, a fluorescent sample of known size (i.e., $20-\mu \mathrm{m}$ fluorescent beads) that is sufficiently larger than the imaging resolution can be used to measure the FOV (Fig. 5a).

\section{Measuring spatial resolution TIMING $1 \mathrm{~h}$}

Measurement of the spatial resolution is recommended to confirm the performance of the fabricated probe. The lateral resolution can be easily measured from the best-focus image of a single $0.2-\mu$ m fluorescent bead. The full-width at half-maximum of the $x$ or $y$ axis intensity profile corresponds to the lateral resolution. The procedure to measure axial resolution is more involved, as described below. The typical lateral and axial resolutions of a GRIN probe are 1 and 10-15 $\mu \mathrm{m}$, respectively. If the measured resolution is substantially higher than these values, the optical probe may need to be replaced.

1. Dilute $0.2-\mu \mathrm{m}$ fluorescent beads in water $(0.1-1 \%$, vol/vol), dispense $10 \mu \mathrm{l}$ on a coverslip and air-dry the coverslip for 20 min.

2. Position the dried sample on the focal plane of the optical probe (Fig. 5 b); refer to Step 24 of the main PROCEDURE.

3. Move the sample away from the focus until fluorescence is not detectable.

4. Take the $z$-stack images of fluorescent beads with steps of $0.5-1 \mu \mathrm{m}$ until no fluorescence is detected. Typically, $z$-axis scanning of $20 \mu \mathrm{m}$ is enough.

$\triangle$ CRITICAL STEP Minimal laser power should be used so as to avoid photobleaching of the fluorescent dye.

5. Plot the intensity over the $z$-axis and measure the full-width at half-maximum of the intensity profile, which is defined as the axial resolution.

\section{Characterizing and compensating chromatic aberration $\bigcirc$ TIMING $1 \mathrm{~h}$}

It is important to understand the chromatic aberration in the GRIN probe in multicolor fluorescence imaging, because the focal shift between different excitation wavelengths must be compensated ${ }^{11}$. To compensate for the chromatic focal shift, the focal position of the objective lens should be adjusted when changing the excitation wavelength. Best-focus images taken at each excitation wavelength at different positions of the objective lens are merged into a single composite image. Typically, the focal planes for two excitation wavelengths of 491 and $532 \mathrm{~nm}$ differ by approximately 20-40 $\mu \mathrm{m}$ depending on the types and lengths of the GRIN lenses. The procedure for characterizing and compensating the focal shift is as follows:

1. Dilute $4-\mu \mathrm{m}$ multicolor fluorescent beads in water (0.1-1\%, vol/vol), dispense $10 \mu \mathrm{l}$ on a coverslip and air-dry the coverslip for $20 \mathrm{~min}$.

2. Position the dried sample on the focal plane of the optical probe.

3. Record the first focal position of the objective lens for the sharpest and brightest image in the 'green' fluorescence channel (Fig. 5c).

4. Record the second focal position of the objective lens for the sharpest and brightest image in the red fluorescence channel (Fig. 5d).

5. The difference between the first and second focal positions is caused by the shift of the focal plane as a result of chromatic aberration of the optical probe, and it represents the chromatic focal shift between the two wavelengths. Merge the green- and red-channel images obtained at the first and second focal positions, respectively, to obtain an aberration-compensated two-color image (Fig. 5e). 
Figure 5 | Optical characterization of a fabricated probe. (a) Measuring the FOV. The image shows fluorescent beads of $20 \mu \mathrm{m}$ in diameter taken with a front-view probe. (b) Measuring spatial resolution. The image shows fluorescent beads of $0.2 \mu \mathrm{m}$ in diameter taken with a frontview probe. (c-e) Characterizing chromatic aberration. The images are fluorescence images of $4-\mu \mathrm{m}$ multicolor beads taken with a front-view probe. Two excitation lasers at 491 and $531 \mathrm{~nm}$ were used, and the emitted fluorescence was collected in the green and red channels.

(c) Two-color fluorescence image when the position of multicolor beads was adjusted to the focus of the 491-nm-excitation light. (d) Two-color fluorescence image when the position of multicolor beads was adjusted to the focus of the 532-nm-excitation light. (e) An aberration-corrected image by merging the green-channel image in $\mathbf{c}$ and the red-channel image in $\mathbf{d}$. Scale bars, $50 \mu \mathrm{m}$.

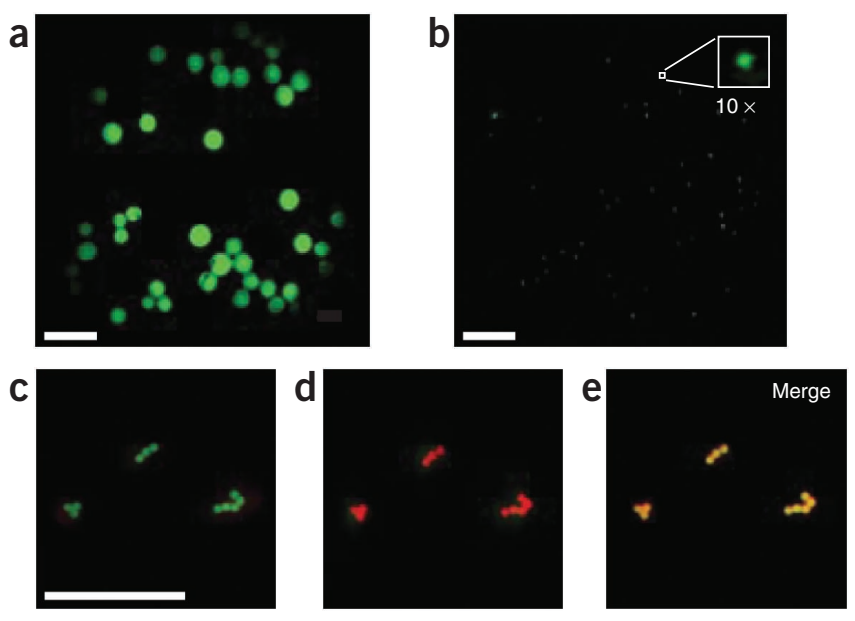

\section{MATERIALS}

\section{REAGENTS}

- UV-curable epoxy (Thorlabs, cat. no. NOA81)

- High-temperature epoxy (Thorlabs, cat. no. 353 NDPK)

- Epoxy mixing kit (Thorlabs, cat. no. EMK 100)

- Hypodermic needle (31 gauge; Myco Medical, cat. no. MEDC-024566)

- Methanol (Fisher Scientific, cat. no. AC32790-0010)

- Ethanol

- Eye ointment (e.g., Opticlox)

- Distilled water

- Mice of the preferred mouse strain (7-16 weeks old). In this protocol, we used wild-type C57BL/6 mice (Jackson Laboratory, C57BL/6J), major histocompatibility complex class II (MHCII)-GFP mice (a gift from H. Ploegh, Whitehead Institute) and Tie2-GFP mice (Jackson Laboratory, stock no. 003658) $\Delta$ CRITICAL All animal experiments must be conducted in accordance with the relevant ethics guidelines and regulations.

- Ketamine (Ketanest, $100 \mathrm{mg} \mathrm{ml}^{-1}$, Pfizer)

- Xylazine (Rompun 2\% (wt/vol), Bayer Healthcare)

- Buprenorphine (Reckitt Benckiser Pharmaceuticals)

- Fluorescent microspheres (10-20 $\mu \mathrm{m}$; Duke Scientific, cat. no. G1000B)

- Multicolor fluorescent microspheres ( $4 \mu \mathrm{m}$; Invitrogen, cat. no. T7284; alternatively F36909)
- Fluorescent microspheres (0.2 $\mu \mathrm{m}$ nm; Duke Scientific, cat. no. G200, and R200)

- Syringe filter (pore size $0.45 \mu \mathrm{m}$; Fisher Scientific, cat. no. 09-720-4)

- Luer lock syringe (10 ml; Air-Tite, cat. no. RL10)

- Tetramethylrhodamine (TAMRA)-dextran conjugate (average mol. wt. 2,000,000 Da; Invitrogen, cat. no. D7139)

- Fluorescein isothiocyanate (FITC)-dextran (average mol. wt. 2,000,000

Da; Sigma-Aldrich, cat. no. FD2000S)

- Nylon suture (Ethilon 6-0)

- Polishing film (Thorlabs, cat. no. LFG1P and LFG5P)

- Saline

EQUIPMENT

Fabrication of optical probes

- GRIN lens (GoFoton, formerly NSG America; IL: cat. no. ILH-100, ILW-100 and RL: SRL-100) or, alternatively, custom probes (GRINTECH)

- Prism mirror (Precision Optics, cat. no. 8531-601; aluminum coating)

- Stainless steel sleeve (NSG America; inner diameter $\sim 1.02 \mathrm{~mm}$, outer diameter $1.25 \mathrm{~mm})$

- Stereoscope (Scienscope, cat. no. NZ-BD-T3)

- Fiber illuminator (Amscope, cat. no. HL250-AY)

- Polishing pad (Thorlabs, cat. no. CTG913)

\section{Box 3 Integration of the probe into a commercial microscope TIMING 1-4 h}

The integration of a GRIN optical probe into an existing microscope system can be achieved with minimal modification of Steps $23-25$ of the main PROCEDURE. Here we describe two integration approaches onto a standard upright microscope system. Briefly, a front-view probe can be held in place using a micro-V clamp mounted on an $x y z$ stage. In contrast, a conventional upright or inverted microscope can be modified to install a side-view probe by using a probe-mount adapter using a simple $4 f$ lens telescope.

\section{Integration of front-view probe TIMING $1 \mathrm{~h}$}

As illustrated in Figure $\mathbf{6 a}$, a micro-V clamp is mounted to a three-axis motorized translation stage using a standard $1 / 2-i n c h$ post and $1 / 2$-inch post holder. A front-view optical probe is held in place with a micro V-clamp. Alternatively, a manual stage can also be used to hold the micro V-clamp.

\section{Integration of side-view probe TIMING $4 \mathrm{~h}$}

An additional side-view imaging path can be built to accommodate the associated relay optics. The purpose of the side-view probe mount is to flip the conjugate scanning plane, located close to the commercial objective mounts, by $90^{\circ}$ and relay it onto the back aperture of a microscope objective. Figure 6 b illustrates a schematic of the adapter for an upright microscope. A silver mirror is used to reflect light exiting the microscope mount. The conjugate scanning plane is relayed onto the back aperture of the microscope objective with a $4 f$ lens system. The first lens is placed one focal length away from the scanning plane. The second lens is placed two focal lengths away from the first lens. The back aperture of the microscope objective is placed one focal length away from second lens. The same adapter can be used for an inverted microscope (not shown). Figure $6 \mathbf{c}$ shows a detailed layout of the mount assembly, much of which is identical to the one shown in Figure $\mathbf{4 c}$. Figure $\mathbf{6 d}$ shows the complete assembly of the side-view probe in an upright microscope. 
Figure 6 | Integration of the probe into a commercial microscope.

(a) A photograph showing a front-view probe mounted on a commercial upright microscope. A micro-V clamp serves as a probe holder.

(b) A schematic illustration for converting an upright imaging path to a side-view imaging path. A mirror reflects the beam by $90^{\circ}$, and two lenses aligned to form a $4 f$ system relays the back aperture beam. (c) A 3D drawing of the side-view mount for a commercial upright microscope. The labels for individual components are consistent with those in Figure 4c. (d) A photograph of the complete setup.

- Polishing disc (Thorlabs, cat. no. D50-SMA)

- Ferrule (Thorlabs, cat. no. 11040A)

- Multipurpose V-groove clamp (Thorlabs, cat. no. HFF001)

- Lock-down clamps (Thorlabs, cat. no. AMA010)

- Fixed angle brackets (Thorlabs, cat. no. AMA009)

- Cross-action tweezers (Roboz, cat. nos. RS5020, RS5027)

- Caliper (Thorlabs, cat. no. CPM1)

- Needles (BD Medical, cat. no. 328418)

- Kimwipes (Thorlabs, cat. no. KW32)

- Lens cleaning tissue (Thorlabs, cat. no. MC-5)

- Dusting kit (Thorlabs, cat. no. CA1)

- UV-curing lamp (Thorlabs, cat. no. CS410-EC)

- Heating pad (Barnstead, cat. no. HP130915)

- Glass-bead sterilizer (Dent-EQ, BS-500)

- Clipper

Probe holder

- Front-view probe holder (Eastern Tools; Supplementary Fig. 2)

- Side-view probe holder (Eastern Tools; Supplementary Fig. 3)

Probe mount

- Single-axis translation stage (Newport, cat. no. Gothic-Arch X)

- $x y$ translation stage (Thorlabs, cat. no. HPT1)

- Cage mounting adapter (Thorlabs, cat. no. CP02B)

- Cage assembly rod (1 inch long, diameter $6 \mathrm{~mm}$; Thorlabs, cat. no. ER1)

- Cage cube (30 mm; Thorlabs, cat. no. C4W)

- Adapter with external SM1 threads and internal RMS threads

(Thorlabs, cat. no. SM1A3)

- Rotation pulley (SDP/SI, cat. no. A6A51)

- Timing belt (SDP/SI, cat. no. A6R51M)

- Ball bearing (SDP/SI, cat. no. A7T55-FS5031)

- Shaft collars (SDP/SI, cat. no. A7X2-13218)

- Rotation shaft (SDP/SI, cat. no. S4012Y-US2-29)

Probe mount attachment

- Assembled probe mount (front view and side view)

- Microscope objective (magnification: $\times 40$, NA: 0.6, dry; Olympus, LUCPlanF1)

- Cage plate (Thorlabs, cat. no. CP02)

- Cage assembly rod (Thorlabs, cat. no. ER1; 1 inch long, diameter $6 \mathrm{~mm}$ )

- Cage cube (30 mm; Thorlabs, cat. no. C4W)

- Post (Thorlabs, cat. no. TR series)

- Linear translation cage mount (Thorlabs, cat. no. SM1Z)

Imaging system

- A confocal microscope system. Either a replica of a custom-designed confocal microscope $\mathrm{e}^{2,3}$ or a commercial upright confocal or two-photon microscope (e.g., Zeiss, Axio Examiner; Thorlabs, multiphoton microscope; Prairie Technology, Ultima multiphoton microscope; LaVision BioTec, TriM scope II) can be used. $\triangle$ CRITICAL The microscope should have sufficient work space under its objective lens, more than $7-10 \mathrm{~cm}$, to accommodate the probe mount and animal stage. If the space is insufficient, the sample stage of the microscope needs to be modified (i.e., LaVision BioTec, Intravital microscope stage).

- Silicone rubber heating sheet (Jxsingtai Electrical)

- $x y z$-axis motorized stage and controller (Sutter Instruments, cat. no. MP285)

- Continuous-wave lasers (Cobalt Laser)

- Silver mirrors and dichroic splitters (Edmund Optics, Chroma Technology)

- Silver-coated polygon scanner (Lincoln Laser)

- Galvanometer (Cambridge Technology)

- Photomultiplier tubes (Hamamatsu, R3896)

- Eight-bit four-channel frame grabber (Matrox Solios eA/XA)

(Optional) Probe mount for commercial microscope

- xyz stage (Sutter Instruments, cat. no. MP285 motorized; or Thorlabs, cat. no. PT3 manual)

- Micro V-clamp (Thorlabs, cat. no. VK250) a

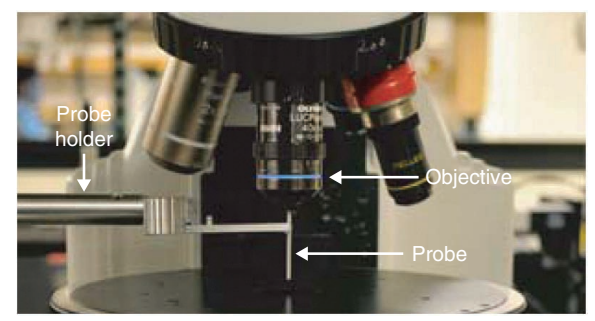

b

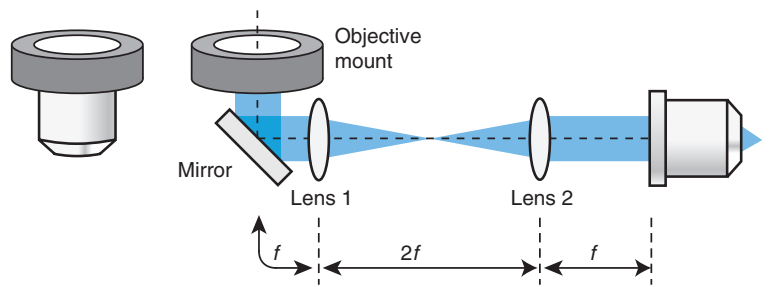

C
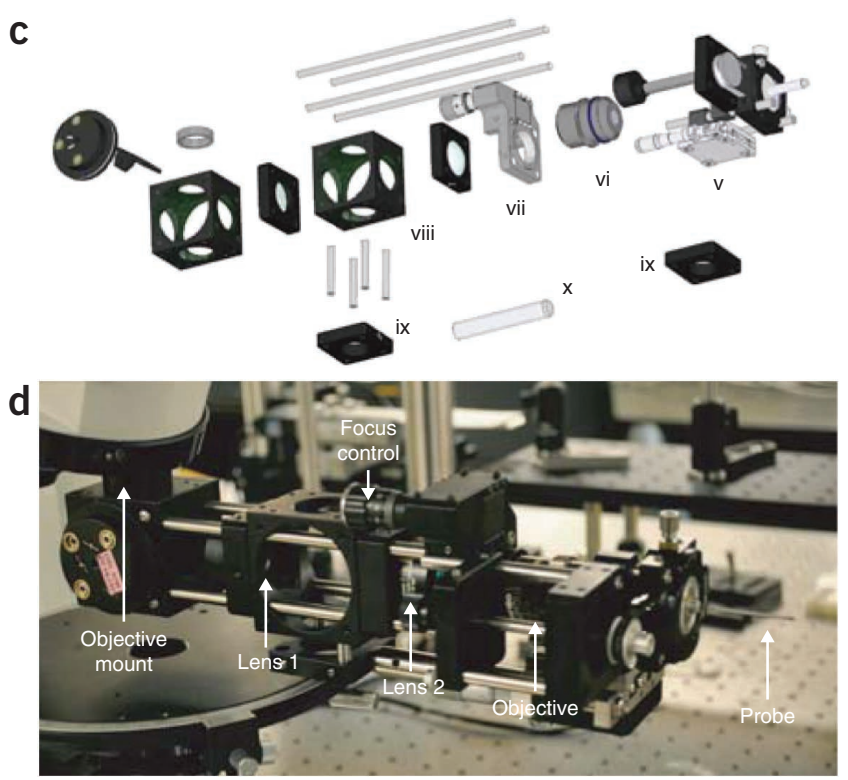

- Adapter with external SM1 threads and internal RMS threads (Thorlabs, cat. no. SM1A3)

- Cage plate (Thorlabs, cat. no. CP02)

- Cage assembly rod (Thorlabs, cat. no. ER1; 1 inch long, diameter $6 \mathrm{~mm}$ )

- Cage cube (30 mm; Thorlabs, cat. no. C4W)

- Achromat lenses (Thorlabs, Edmund optics and so on)

REAGENT SETUP

Animal housing Before imaging, the mouse should be housed in a cage with free access to food and water. The cage should have proper temperature/humidity control, ventilation and regular cleaning. After imaging, the mouse should be kept in a warm environment and observed until it recovers from anesthesia. Ketamine/xylazine solution Mix $1 \mathrm{ml}$ of ketamine $\left(100 \mathrm{mg} \mathrm{ml}^{-1}\right), 0.15 \mathrm{ml}$ of xylazine $\left(100 \mathrm{mg} \mathrm{ml}^{-1}\right)$ and $8 \mathrm{ml}$ of sterile saline under aseptic conditions. The mixture volume can be modified depending on the number and weight of mice. $\Delta$ CRITICAL The ketamine/xylazine solution should be prepared and used on the same day.

Fluorescent dyes Dissolve the FITC-dextran or TAMRA-dextran in sterile $5 \%(\mathrm{wt} / \mathrm{vol})$ saline and filter the solution through a $0.45-\mu \mathrm{m}$ syringe filter. The solution can be aliquotted $(100 \mu \mathrm{l})$ and stored in lightproof vials for future use. The typical maximum storage time is 1 month at $-20^{\circ} \mathrm{C}$ and 6 months at $-80^{\circ} \mathrm{C}$.

High-temperature epoxy Mix the thixotropic epoxy (pack A) and the hightemperature epoxy (pack B) on an aluminum tray. $\Delta$ CRITICAL The mixture should be prepared and used on the same day, just before attaching the metal sleeve (Step 21), because epoxy polymerization curing starts even at room temperature $\left(18-20^{\circ} \mathrm{C}\right)$. 


\section{EQUIPMENT SETUP}

Metal sleeve A stainless steel sleeve with an inner diameter of $1.02 \mathrm{~mm}$ and an outer diameter of $1.25 \mathrm{~mm}$ is used as a metal sleeve to protect the adjoining points of the optical probe during routine handling and imaging. The metal sleeve needs to be cut and trimmed to the desired length to adequately cover the adjoining points of the fabricated optical probe without exceeding its total length. The metal sleeve can be prepared with a grinding machine. Alternatively, commercial metal cutting services are available from GRINTECH or local machine shops. ! CAUTION Care is necessary to avoid physical injury during the cutting and grinding of the metal tube.

Jig After removing the plastic part of the SMA connector (Supplementary

Fig. 1), connect the polishing disc with the SMA connector, which has a hole diameter of $1.03 \mathrm{~mm}$.

Optical probe Before imaging, sterilize optical probes with $100 \%$ ethanol solution.

Front-view probe holder A custom-made stainless disc (25.4 mm diameter) disc with a hole (1.26 $\mathrm{mm}$ diameter) in the center is used for holding the optical probe (Supplementary Fig. 2).

Side-view probe holder A custom-made stainless tube $(4.28 \mathrm{~mm}$ outer diameter, $1.2 \mathrm{~mm}$ inner diameter) used for holding the optical probe is inserted into a ball bearing that is already glued to a custom-made stainless bearing collar. A rotation pulley is attached to the tube using a set screw (Supplementary Fig. 3). $\Delta$ CRITICAL Owing to its small size, the optical probe must be handled with care during insertion into the holder via the thru-hole. The outer diameter of the optical probe holder is matched to the internal thread of the $x y$ translation stage (Fig. 3b).
Front-view probe mount An $x y$ translation stage is mounted onto a singleaxis translation stage using two assembly cage rods and a cage adapter mount, as shown in Figure 3a.

Side-view probe mount A probe mount is designed to provide rotation and translation of a side-view optical probe, as shown in Figure 4a. The detailed procedure for assembling the attachment mount is illustrated in Supplementary Figure 4. Briefly, a probe holder composed of a rotation pulley is attached to an $x y$-translation cage mount. A rotation shaft is connected with a timing belt, a cage mounting adapter and a cage assembly rod to the probe holder and the $x y$-translation cage mount. The assembled mount is attached to a single-axis translation stage with two cage rods and a cage adapter mount (Fig. $\mathbf{4 b}, \mathbf{c}$ ).

Assembly of objective lens mount The microscope objective and the probe mount are attached to a cage cube (Figs. $3 \mathrm{c}$ and $\mathbf{4 c}$ ). The microscope objective has an independent focus control, $z$-translation stage, which is used to manually shift the focal plane of the objective.

Custom confocal microscope The imaging system is a custom-built videorate scanning laser confocal microscope ${ }^{11}$. The system uses continuous-wave lasers at 491, 532 and $635 \mathrm{~nm}$ for single-photon fluorescence excitation. Silver mirrors and dichroic splitters were used to select and direct a laser beam to a raster beam scanner comprising a silver-coated polygon scanner and a galvanometer. The microscope was designed to have a FOV of 250-300 $\mu \mathrm{m}$ when an $\times 40$ objective lens is used. Three photomultiplier tubes (PMTs) were used to collect fluorescence signals in three fluorescence channels, respectively. An 8-bit four-channel frame grabber digitizes the PMT signals at 20 megasamples per second for $512 \times 512$ pixels per frame. Customized software is used to display and save the acquired images at a frame rate of $15-30 \mathrm{~Hz}$ in real time.

\section{PROCEDURE}

CL assembly $\bigcirc$ TIMING 30 min

1| Mount an RL on a V-groove clamp by using a pair of tweezers (Fig. 2a,b).

2| Adjust the focus of a top-viewing stereoscope and a side-viewing stereoscope to coincide at the top surface of the mounted RL.

3| Partly dip the tip of a 31-G syringe needle into the UV-curable epoxy and extract a small droplet of UV epoxy.

4| Apply $\sim 1 \mu \mathrm{l}$ of UV-curable epoxy over the central portion of the RL surface with visual inspection through the stereoscopes by carefully touching only the epoxy droplet at the syringe needle tip with the RL surface (Fig. 2c).

! CAUTION Epoxy can cause irritation of the skin. Wear protective gloves when handling epoxy.

$\triangle$ CRITICAL STEP Be careful not to damage the RL surface with the syringe needle. This step can be done manually by a skilled experimenter. An xyz micrometer stage with a mounted syringe tip can be helpful for an unskilled experimenter. In addition, an excessive amount of epoxy can cause a tilt at the lens interface in the next step (Step 5). If excessive epoxy is applied, a lens cleansing tissue soaked with 100\% methanol can be used to remove the remaining epoxy, and then Steps 3 and 4 can be repeated. Ensure that there is no remaining epoxy or dust on the RL surface through a top-viewing stereoscope before repeating Step 3.

5| Place a $\mathrm{CL}$ on top of the RL, using a pair of tweezers, and look through the stereoscopes to ensure proper alignment of the $\mathrm{CL}$ and the RL (Fig. 2c).

$\Delta$ CRITICAL STEP This step can markedly affect the imaging performance of an optical probe. It is recommended to achieve alignment of the lenses within $30 \mu \mathrm{m}$ by using the stereoscopes. If misalignment is observed, correct the displacement by gently touching the $\mathrm{CL}$ using a tweezer. If tilt is observed, clean the surfaces of lenses using a lens cleansing tissue soaked with $100 \%$ methanol and restart from Step 3 with a reduced amount of epoxy.

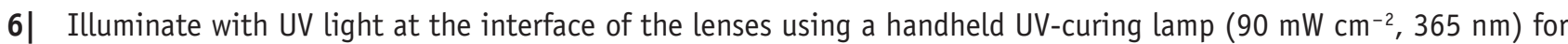
5 min. For front-view optical probes, proceed directly to Step 15. For side-view probes, continue with Step 7.

! CAUTION Wear UV protection goggles. Avoid direct exposure of UV light to the experimenter, as UV light can seriously damage the retina and skin.

? TROUBLESHOOTING 


\section{Polishing (for side-view optical probe only) $\bigcirc$ TIMING $1 \mathrm{~h}$}

7| Spray distilled water on the polishing pad. Two or three pumps from a spray bottle are adequate.

8| Place the $5-\mu \mathrm{m}$ polishing film on the wet polishing pad. The polishing film should adhere firmly to the polishing pad.

9| Apply enough distilled water to the polishing film to soak the whole film, and then remove excess water with a clean tissue.

10| Place a polishing disc on the wet polishing film and insert the IL, using a pair of tweezers, into the ferrule hole in the polishing disc (Supplementary Fig. 1).

11 Insert a pushpin into the same ferrule hole and on top of the IL. This is to ensure that the GRIN lens stays in place and in contact with the polishing film during the polishing process.

12| Polish the IL by sliding the polishing disc 20-50 times along a path that resembles the Arabic number 8 , and then measure the changes to the length of the IL using a caliper.

$\triangle$ CRITICAL STEP Be careful not to damage the surface of the IL during length measurement with the caliper.

13| Repeat Steps 10-12 until the IL is about $0.1 \mathrm{~mm}$ longer than the desired length (e.g., $1.8 \mathrm{~mm}$ for the desired length of $1.7 \mathrm{~mm}$ ).

$\triangle$ CRITICAL STEP Be careful not to polish beyond the desired length. Frequent length measurement is recommended for an unskilled experimenter.

14| Repeat Steps 7-13 with a 1- $\mu \mathrm{m}$ polishing film until the desired length is achieved. Inspect the surface quality and flatness by using stereoscopes.

$\triangle$ CRITICAL STEP This step can markedly affect the imaging quality. Check with the stereoscopes that the surface is flat and clean. A lens cleansing tissue soaked with $100 \%$ methanol can be used to clean the lens surface.

? TROUBLESHOOTING

\section{IL assembly $\bigcirc$ TIMING 30 min}

15| Flip the RL with a pair of tweezers and mount it on a V-groove clamp.

16| Repeat Steps 2-6 with the IL. For front-view optical probes, continue from Step 20 of the PROCEDURE. For side-view optical probes, proceed to Step 17.

\section{Prism mirror assembly (for side-view optical probe only) TIMING 30 min}

17| Repeat Step 3 and apply $\sim \mu$ l of UV-curable epoxy over the central portion of the IL surface, with visual inspection through stereoscopes, by carefully touching only the epoxy droplet at the syringe needle tip with the RL surface (Fig. 2f).

18| Hold the triangular sides of the prism using a pair of tweezers and place it on top of the IL.

19| Align the prism to fit inside the circular face of the IL by gently touching the prism mirror using a pair of tweezers with visual feedback through a front-viewing stereoscope, and then repeat Step 6.

$\triangle$ CRITICAL STEP Be careful not to damage the rectangular sides of the prism. Excessive glue should be removed with a lens cleaning tissue, as it can hinder insertion into the metal sleeve (Step 20).

\section{Metal sleeve $\bigcirc$ TIMING $3 \mathrm{~h}$}

20| Insert the assembled optical probe into the prepared metal sleeve (see EQUIPMENT SETUP). The assembled optical probe should smoothly slide into the metal sleeve without resistance.

? TROUBLESHOOTING

21| Dip the tip of a syringe needle into high-temperature epoxy and use the epoxy to fill the gap between the inserted optical probe and the metal sleeve. For a side-view optical probe, seal the prism edge with the epoxy to form a smooth surface (Fig. 2j).

$\triangle$ CRITICAL STEP Be careful not to cover the optical path with the epoxy or to leave epoxy on the outside of the metal sleeve. High-temperature epoxy at the focus of the laser beam can produce strong autofluorescence, resulting in unwanted background noise during imaging. 
22 Keep the sleeved optical probe on the heating pad at $120^{\circ} \mathrm{C}$ for $2 \mathrm{~h}$. This will cure the high-temperature epoxy. ! CAUTION Heating pads can cause burn injuries. Use a pair of tweezers when handling the sleeved optical probe and avoid direct contact of the heating pad with skin.

\section{Integration of the probe into a microscope (front and side view) TIMING $\mathbf{1 ~ h}$}

23 Secure the optical probe on a custom-designed mount with three-axis translation control or an additional rotation control for a side-view optical probe (see Figs. 3 and 4, Equipment Setup, and Supplementary Video 1).

$\triangle$ CRITICAL STEP Insertion and fixing of the probe in the holder should be carried out with great care, because bending or stress can cause damage to the epoxy glue between the GRIN lenses or cracking of the lens itself.

24 Adjust the proximal surface of a $\mathrm{CL}$ of the optical probe to a position coincident with the focal plane of a microscope's objective lens (Supplementary Video 2).

$\triangle$ CRITICAL STEP This step requires precision positioning of the optical probe relative to the objective by adjusting the probe mount and $z$-axis translational stage. We recommend using the fluorescence signal as feedback for alignment. Drop a small solution of large fluorescent beads (10-20 $\mu \mathrm{m}$ diameter) onto the distal surface. Adjust the optical probe mount to maximize the fluorescent signal. Once this position is established, wash the beads off the probe surface with physiological saline. Detailed characterization of probe performance can be found in Box 2. In our custom confocal microscope, we can also remove an emission filter temporarily and image the optical probe surfaces using the reflected excitation light as feedback for probe alignment.

! CAUTION Laser light can cause serious damage to the retina. Wear protective goggles and avoid direct eye exposure to the laser. ? TROUBLESHOOTING

25 Adjust the focus control knob such that the focal plane of the objective lens is placed inside the optical probe at a depth of 30-50 $\mu \mathrm{m}$ from the proximal surface. It will place the imaging plane of the GRIN probe at about 30-50 $\mu \mathrm{m}$ outside the distal surface of the probe in air (or at 20-35 $\mu \mathrm{m}$ in a sample with a refractive index of 1.5). This setting helps the user determine whether the probe is in contact with the sample or tissue by looking at the images.

\section{Mouse preparation $\bigcirc$ TIMING $30 \mathrm{~min}$}

26| Anesthetize the mouse using an intraperitoneal injection of ketamine/xylazine solution $\left(75 \mathrm{mg} \mathrm{kg}^{-1}\right.$ for ketamine and $15 \mathrm{mg} \mathrm{kg}^{-1}$ for xylazine) and wait for 2-3 min until the mouse is sufficiently anesthetized.

$\triangle$ CRITICAL STEP All animal experiments must be conducted in accordance with the guidelines and regulations of the relevant authorities.

$\Delta$ CRITICAL STEP Monitor the depth of anesthesia by checking reflexes (i.e., toe pinching, eyelid blinking), respiration and heart rate.

27| Apply eye ointment to the eyes of the mouse to prevent corneal damage.

28| Remove the hair around the target area using clippers and hair removal cream.

29| If necessary, make a 3- to 5-mm incision on the skin and peritoneum. This step is not required if the optical probe is inserted through a natural opening, such as the colon.

30| Irrigate the target organ with a $37^{\circ} \mathrm{C}$ physiological saline.

$\triangle$ CRITICAL STEP This step is important for washing away natural waste or surgical artifacts (such as blood) and lubricating the target tissue. For colon tissue, irrigate several times by injecting $\sim 0.5 \mathrm{ml}$ of physiological saline with a rubber-tipped syringe, taking care not to induce bleeding.

31 (Optional) If fluorescent labeling of blood vessels is required, inject $100 \mu \mathrm{l}$ of TAMRA-dextran or FITC-dextran $(5 \%(\mathrm{wt} / \mathrm{vol})$ in sterile saline) intravenously, if needed.

\section{Mouse imaging $\bigcirc$ TIMING 1-2 $\mathrm{h}$}

32| Place the mouse on a heated translation stage in the desired position (Figs. $3 \mathbf{d}$ and $\mathbf{4 d}$ ).

33| Move the mouse on the translation stage so that the optical probe can be inserted through the incision or natural opening and reach the target tissue (Supplementary Video 3).

$\triangle$ CRITICAL STEP To avoid tissue damage, such as bleeding, do not press the target tissue with the optical probe. In addition, care should be taken not to bend the optical probe by pushing against the tissue, because it can cause the individual GRIN lenses in the probe to crack or it can damage the epoxy glue between lenses. 
PROTOCOL

34| Adjust laser power and PMT gain until a sufficient signal-to-noise ratio is achieved. Keep the laser power as low as possible to prevent photobleaching and tissue damage.

35 Acquire images or movies with changing focus, position of the mouse stage and rotation (side-view optical probe only; see Supplementary Video 3).

$\Delta$ CRITICAL STEP Monitor the depth of anesthesia by checking reflexes (i.e., toe pinching, eyelid blinking) respiration and heart rate.

? TROUBLESHOOTING

36 If required, close the incision with a nylon suture and administer analgesics such as buprenorphine $\left(0.1 \mathrm{mg} \mathrm{kg}^{-1}\right.$ subcutaneously, every $12 \mathrm{~h}$ for $3 \mathrm{~d}$ ). For side-view imaging through natural openings, there is no need for suturing.

$\triangle$ CRITICAL STEP In the case of longitudinal studies, it is crucial to minimize stress and infection to the animals from repeated anesthesia and surgical intervention.

\section{? TROUBLESHOOTING}

Troubleshooting advice can be found in Table 3.

TABLE 3 | Troubleshooting table.

\begin{tabular}{|c|c|c|c|}
\hline 6 & No epoxy curing & Insufficient UV illumination & $\begin{array}{l}\text { Increase the duration of illumination; place the } \\
\text { UV curing lamp closer }\end{array}$ \\
\hline 14 & Cannot achieve polishing & The weight of the pushpin is insufficient & Increase the weight of the pushpin \\
\hline \multirow[t]{2}{*}{20} & $\begin{array}{l}\text { Cannot insert the optical } \\
\text { probe into metal sleeve }\end{array}$ & The metal sleeve is too small & $\begin{array}{l}\text { Carefully remove excessive UV epoxy from the } \\
\text { GRIN lenses and prism }\end{array}$ \\
\hline & & GRIN lenses are not aligned properly & Replace the probe with a new one \\
\hline \multirow[t]{2}{*}{24} & $\begin{array}{l}\text { Cannot obtain fluorescence/ } \\
\text { reflectance image }\end{array}$ & PMT gain is too low & Increase PMT gain \\
\hline & & The optical probe is out of focus & Adjust the position of optical probe mount \\
\hline \multirow{4}{*}{35} & & $\begin{array}{l}\text { Insufficient fluorescence excitation or } \\
\text { PMT gain is too low }\end{array}$ & Increase laser power or PMT gain \\
\hline & & $\begin{array}{l}\text { Dirt or blood has accumulated on the } \\
\text { optical probe surface }\end{array}$ & $\begin{array}{l}\text { Retract and clean the optical probe surface and } \\
\text { irrigate the tissue with physiological saline }\end{array}$ \\
\hline & & $\begin{array}{l}\text { The concentration of fluorescent dye } \\
\text { is too low }\end{array}$ & Increase concentration of fluorescent dye \\
\hline & & $\begin{array}{l}\text { Broken UV epoxy bonding between } \\
\text { lenses or cracked GRIN lenses }\end{array}$ & Replace the broken probe with a new one \\
\hline
\end{tabular}

TIMING

Steps 1-6, assembling a CL to an RL: 30 min

Steps 7-14, polishing an IL to the designed length (for side-view probe only; inexperienced experimenters may need

more time): $1 \mathrm{~h}$

Steps 15 and 16, assembling an IL to an RL: 30 min

Steps 17-19, assembling a prism mirror (for side-view probe only): 30 min

Steps 20-22, inserting a prepared metal sleeve: $3 \mathrm{~h}$ 
Figure 7 | In vivo fluorescence images obtained by a front-view probe. (a-f) Images of $\mathrm{MHCII}^{+}$, $\mathrm{GFP}^{+}$cells in intestine, spleen, kidney, liver, bladder and ureter, as indicated. Vasculature is visualized by the fluorescence (red) from the TAMRA-dextran conjugates in the blood stream (red). Dendritic-like cells are clearly visible in all images. (g, h) Images of $\mathrm{Tie}^{+}, \mathrm{GFP}^{+}$endothelial cells in kidney and liver, as indicated. Scale bars, $100 \mu \mathrm{m}$.

Steps 23-25, integration of the probe into a microscope: $1 \mathrm{~h}$

Steps 26-31, mouse preparation: $30 \mathrm{~min}$ Steps 32-36, performing mouse imaging: $1-2 \mathrm{~h}$

Box 2, optical characterization of the probe: $2.5 \mathrm{~h}$

Box 3, integration of the probe into a commercial microscope: 1-4 $\mathrm{h}$
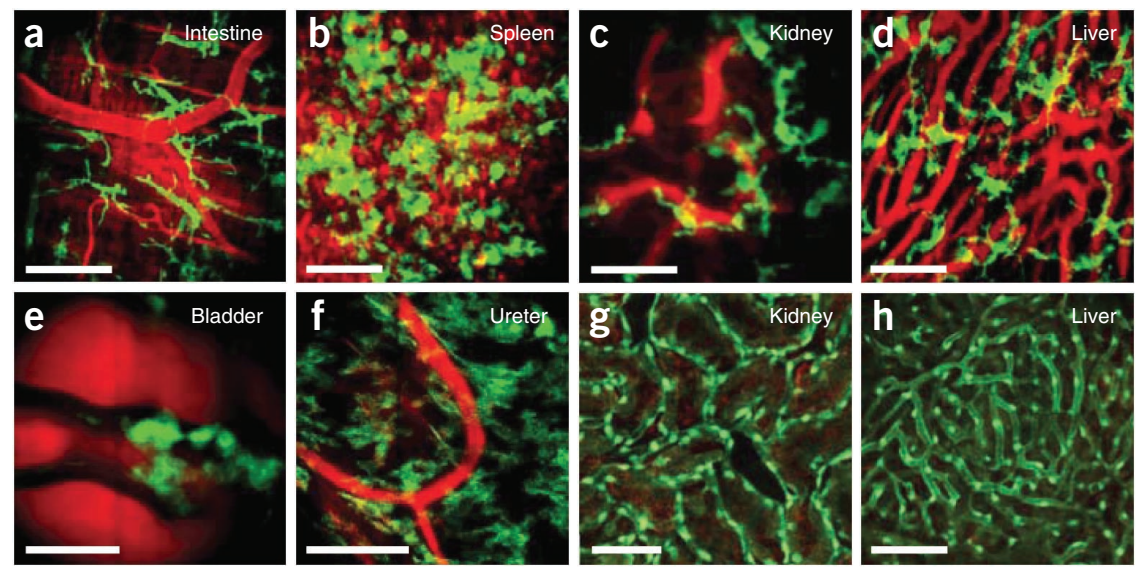

\section{ANTICIPATED RESULTS}

Our research has shown that minimally invasive imaging of internal organs at the cellular level in vivo is a powerful tool for understanding in vivo biology, mechanisms of diseases and the effectiveness of new therapies. Successful completion of this protocol will provide the user with such an imaging system by integrating either a fabricated or purchased GRIN probe into a microscope available to the user. Anticipated results for various steps of the protocol are described here.

Before beginning the fabrication of an optical probe, it is important to determine the optimal design depending on specific target organs. General design and selection guidelines are summarized in Tables 1 and 2 . The principles and components of a front-view and side-view triplet GRIN probes, respectively, are illustrated in Figure 1. Successful completion of the fabrication steps (Fig. 2c-k) will produce a front-view probe with a length of 20-35 mm or a side-view probe with a length of $60-100 \mathrm{~mm}$ as shown in Figure 2i. Completion of the assembly steps illustrated in Figures 3, 4 or $\mathbf{6}$ will result in a fully integrated imaging system based on the optical probes.

The endomicroscopy system based on the front-view probe will allow the user to conduct fluorescence cellular imaging of viscera organs after minimally invasive laparotomy². Figure 7 shows exemplary results obtained with various organs, such as the small intestine, spleen, kidney, liver, bladder and ureter. In Figure 7a-f, we used a transgenic mouse, in which green fluorescent protein (GFP) is expressed under the direction of the MHCII promoter. $\mathrm{MHCII}^{+}, \mathrm{GFP}^{+}$antigen-presenting cells are observed in green, and the vasculature is visualized by intravenously injected dye-dextran conjugates. In Figure $\mathbf{7 g}, \mathbf{h}, \mathrm{a}$ Tie2-GFP transgenic mouse was imaged, in which the endothelial cells of blood vessels are visualized by GFP fluorescence. By minimizing the surgical opening in the imaging procedure, it is possible to perform repeated imaging in a mouse at multiple

time points. To illustrate this possibility, we performed serial imaging of immune cells in a mouse model of renal transplantation ${ }^{12}$. A donor kidney was harvested from a MHCII-GFP mouse in C57BL/6 background strain and then transplanted into a wild-type mouse in BALB/C background strain, and the front-view probe was used to monitor the donor GFP-expressing antigen-presenting cells in the kidney allograft. The tissues in the kidney expressed relatively strong autofluorescence, which visualizes kidney tubules in the cortex. At day 1 after transplantation, a substantial number of dendritic-shaped GFP+ donor APCs are observed (Fig. 8a). At day 3, most dendritic GFP cells have entered the capsules, which indicates the process of clearance (Fig. $\mathbf{8 b}$ ). The level of autofluorescence in the kidney capsule is also indicative of the viability of the tissue, as shown in the controls (Fig. 8c,d) compared with the grafted organs. In addition, time-lapse imaging allows the migration of immune cells to be tracked in the kidney (Supplementary Fig. 5).

The endomicroscopy system with the side-view probe will enable the user to perform noninvasive imaging of the
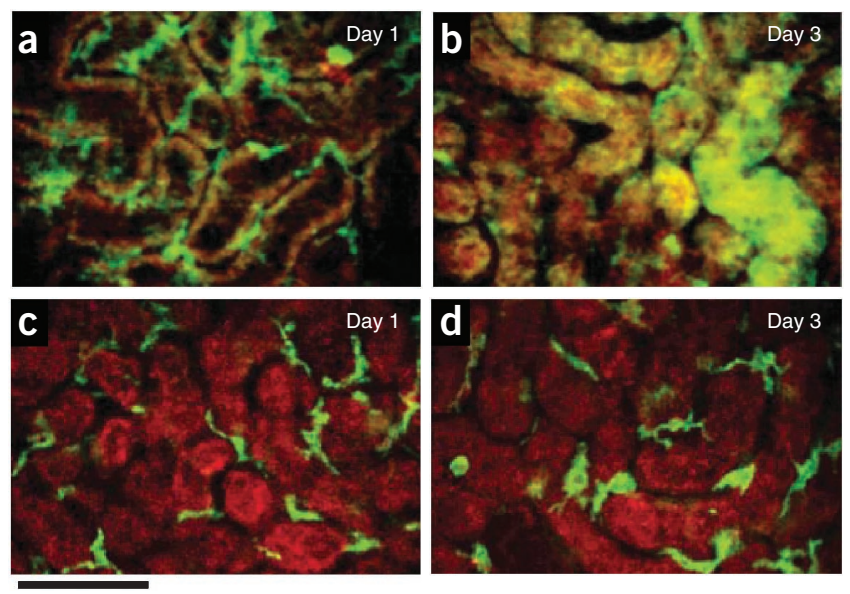

Figure 8 | Time-lapse imaging of kidney graft with front-view probe. $(\mathbf{a}, \mathbf{b})$ Donor-origin $\mathrm{MHCII}^{+}, \mathrm{GFP}^{+}$cells in kidney graft after allogeneic transplantation at day 1 (a) and day 3 (b). Renal tubules are visualized by red autofluorescence. (c,d) Controls. $\mathrm{MHCII}^{+}, \mathrm{GFP}^{+}$cells in the normal kidney at day 1 (c) and day 3 (d) after sham surgery. Scale bar, $100 \mu \mathrm{m}$. 
Figure 9 | In vivo fluorescence images by sideview probe. $(\mathbf{a}-\mathbf{c})$ Single-FOV images taken in the descending colon. (a) $\mathrm{MHCII}^{+}, \mathrm{GFP}^{+}$cells in the colonic mucosa. (b) Epithelial cells stained with systemically delivered Evans blue. (c) Vasculature visualized by intravenously injected FITC-dextran conjugates. (d,e) Largearea images constructed from the mosaic of multiple single-FOV images. (d) Vasculature in the descending colon. (e) A fly-through rendering. Scale bars, $200 \mu \mathrm{m}$.
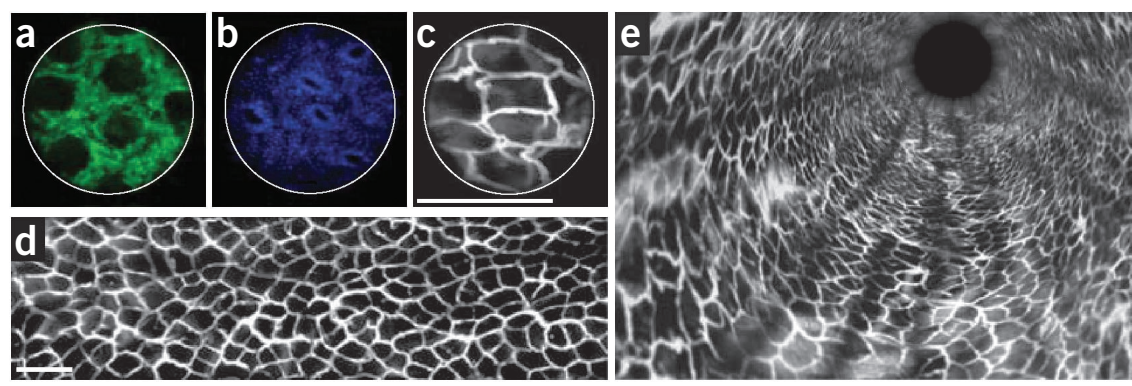

mucosa of tubular organs such as gastrointestinal tracts and respiratory airways through natural orifices ${ }^{3}$. Figure 9 shows exemplary imaging results of the descending colon showing fluorescently labeled cells and blood vessels. Before colon imaging, a mouse is starved for $24 \mathrm{~h}$ to empty the colon, and immediately before imaging the belly of the mouse is gently swept toward the rectum to squeeze out any stool remaining in the colon. The anesthetized mouse is placed on its back on the plate with its anus facing the optical probe and moved slowly toward the probe by the $x y z$ translation stage. By orienting the view window of the optical probe toward the ventral side, the position of the tip of the probe within the colon can be observed. It is helpful to monitor the length of the optical probe inserted into the animal for position registration. The optical probe is rotated and translated along the mucosa using the customized probe mount. Once the optical probe is in place, the user can observe the images on a computer display and record images. Figure $\mathbf{9 a - c}$ shows fluorescence images taken using the side-view optical probe in the normal mucosa of the descending colon, which show $\mathrm{MHCII}^{+}, \mathrm{GFP}^{+}$cells, epithelial cells lining the crypt structure and the blood vasculature visualized by FITC-dextran in the bloodstream. A 3D data set of the vasculature was acquired by scanning the probe over a wide area of the tissue at a speed of $100-200 \mu \mathrm{m} \mathrm{s}^{-1}$. Rotation and registration of individual images allow a large-area mosaic image to be constructed (Fig. 9d) ${ }^{3}$. The data set can be rendered in various modes, such as a fly-through presentation (Fig. 9e). By using a similar procedure, vasculature and cellular images can be obtained from the upper gastrointestinal tracts and respiratory tracts ${ }^{3}$.

The side-view optical probe is well suited to tracking the development of colorectal tumors in a spontaneous colorectal cancer mouse model. We used a genetically modified mouse model, in which the adenomatous polyposis coli ( $A p c)$ gene in the intestinal cells in the colonic mucosa can be knocked out by administration of adenoviral Cre. The inactivation of $A p c$ is followed by the activation of the reporter gene of GFP to enable the long-term tracking and quantification of the growth of Apc-mutated cells in the colon by side-view fluorescence endomicroscopy ${ }^{3}$. By using the side-view optical probe and the imaging procedures described earlier, we observed multiple GFP-expressing polyps at 4 weeks after the viral delivery of Cre (Fig. 10a). Blood vessels are simultaneously visualized by intravenously injected TAMRA-dextran. At this stage, two micronodules with diameters of 50-150 $\mu \mathrm{m}$ are detected, in which noticeable changes in vasculature are observed. Two weeks later, the same area can be identified at approximately the same distance from the anus, and it is found that one of the nodules has grown to approximately $400 \mu \mathrm{m}$ in diameter (Fig. 10b). Dilation of blood vessels surrounding the nodules is clearly observed. The injected dye can leak out of the permeable vessels in tumors, which makes it difficult to image the vasculature repeatedly at a short time interval.
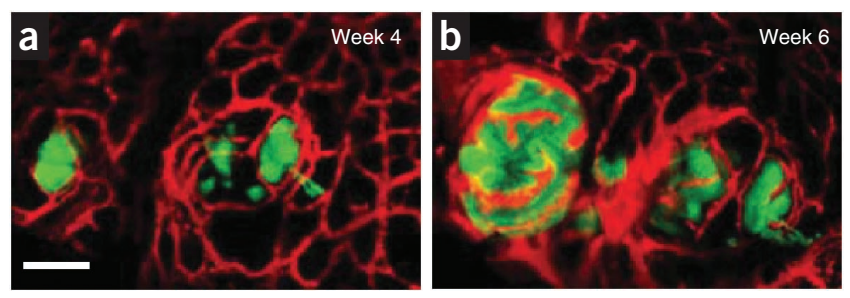

Figure 10 | Time-lapse imaging of colorectal tumorigenesis with side-view probe. (a) $\mathrm{GFP}^{+}$polyps and vasculature at 4 weeks after $A p c$ inactivation by delivery of adenoviral Cre into the colon. (b) The same region in a at 6 weeks. Scale bar, $100 \mu \mathrm{m}$.
Note: Supplementary information is available in the online version of the paper.

ACKNOWLEDGMENTS We thank R. Colvin and C. Chase for providing the mice with renal transplantation; R. Kucherlapati and K. Hung for the mouse model of spontaneous colorectal tumor; H. Ploegh for the MHCII-GFP mice; C. Lin, D. Cote, L. Kaplan, M. Ferrari and G.Y. Koh for discussions; and D. Stevenson for proofreading of the manuscript. This work was supported by grants from the US National Institutes of Health (R21AI081010, RC1DK086242, RC2DK088661, U54CA143837, R01AI081734, P41EB015903), the US Department of Defense (FA9550-10-1-0537) and the National Research Foundation of Korea (WCU R31-2008-000-10071-0, 2009-352-C00042, 2011-0009503, WCI 2011-001).

AUTHOR CONTRIBUTIONS J.K.K., W.M.L., P.K., M.C. and S.H.Y. designed and wrote the protocol. K.J. and S.K. contributed figures.
COMPETING FINANTIAL INTERESTS The authors declare no competing financial interests.

Published online at http://www.nature.com/doifinder/10.1038/nprot.2012.078 Reprints and permissions information is available online at http://www.nature. com/reprints/index.html.

1. Flusberg, B.A. et al. Fiber-optic fluorescence imaging. Nat. Meth. 2, 941-950 (2005).

2. Fan, Z. et al. In vivo tracking of 'color-coded' effector, natural and induced regulatory $\mathrm{T}$ cells in the allograft response. Nat. Med. 16, 718-722 (2010).

3. Kim, P. et al. In vivo wide-area cellular imaging by side-view endomicroscopy. Nat. Meth. 7, 303-305 (2010). 
4. Kiesslich, R., Goetz, M., Vieth, M., Galle, P.R. \& Neurath, M.F. Technology insight: confocal laser endoscopy for in vivo diagnosis of colorectal cancer. Nat. Clin. Pract. Oncol. 4, 480-490 (2007).

5. Becker, C., Fantini, M.C. \& Neurath, M.F. High resolution colonoscopy in live mice. Nat. Protoc. 1, 2900-2904 (2006).

6. Hsiung, P.L. et al. Detection of colonic dysplasia in vivo using a targeted heptapeptide and confocal microendoscopy. Nat. Med. 14, 454-458 (2008).

7. Dela Cruz, J.M., McMullen, J.D., Williams, R.M. \& Zipfel, W.R. Feasibility of using multiphoton excited tissue autofluorescence for in vivo human histopathology. Biomed. Opt. Express 1, 1320-1330 (2010).

8. Waldner, M.J., Wirtz, S., Neufert, C., Becker, C. \& Neurath, M.F. Confocal laser endomicroscopy and narrow-band imaging-aided endoscopy for in vivo imaging of colitis and colon cancer in mice. Nat. Protoc. 6 , 1471-1481 (2011).
9. Barretto, R.P. et al. Time-lapse imaging of disease progression in deep brain areas using fluorescence microendoscopy. Nat. Med. 17, 223-228 (2011).

10. Jung, J.C. \& Schnitzer, M.J. Multiphoton endoscopy. Opt. Lett. 28, 902-904 (2003).

11. Kim, P., Puoris'haag, M., Cote, D., Lin, C.P. \& Yun, S.H. In vivo confocal and multiphoton microendoscopy. J. Biomed. Optics 13, 010501 (2008).

12. Miyajima, M. et al. Early acceptance of renal allografts in mice is dependent on Foxp3(+) cells. Am. J. Pathol. 178, 1635-1645 (2011).

13. Cole, R.W., Jinadasa, T. \& Brown, C.M. Measuring and interpreting point spread functions to determine confocal microscope resolution and ensure quality control. Nat. Protoc. 6, 1929-1941 (2011). 